\title{
Pre-analytical rejection rates of clinical samples based on patients' health status
}

\section{Adem Keskin ${ }^{1}\left([)\right.$ and Recai $A_{c i}^{2}{ }^{(0)}$}

${ }^{1}$ Department of Biochemistry, Institute of Health Sciences, Aydin Adnan Menderes University, Aydin, Turkey

${ }^{2}$ Department of Biochemistry, Samsun Training and Research Hospital, Health Sciences University, Samsun, Turkey

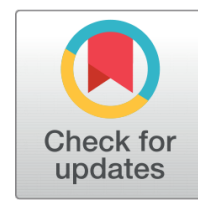

Received 10-01-2022
Revised 23-02-2022
Accepted 24-02-2022
Published 27-02-2022

Corresponding Author Adem Keskin

ademkeskin78@gmail.com

Department of Biochemistry, Institute of Health Sciences, Aydin Adnan Menderes University, Aydin, Turkey

DOI https://doi.org/10.47419/ bjbabs.v3i01.94

Pages: 29-39

Distributed under the terms of the Creative Commons

Attribution-NonCommercial 4.0 International (CC-BY-NC 4.0), which permits use for any non-commercial purpose, distribution, and reproduction in any medium, provided that the original work is properly cited.

Copyright: (c) 2022 Adem

Keskin, Recai Aci

\section{OPEN ACCESS}

\section{ABSTRACT}

Background and objective : The pre-analytical rejection rate is the proportion of samples rejected at the stage that includes the initial procedures of the testing process performed outside the laboratory walls by healthcare professionals. This study aimed to evaluate the pre-analytical rejection rate by considering the health status of the patients and the sample types and to examine the measures that can be taken against it.

Methods: The data of the samples that came to the laboratory for analysis for one year were included. These data were categorized according to sample types in complete blood count, biochemistry, hormones, urine, blood gases, coagulation, erythrocyte sedimentation rate (ESR), glycosylated hemoglobin $\left(\mathrm{HbA}_{1} c\right)$. It was also categorized by emergency, outpatient, inpatient, and critically ill status. Considering the health status of the patients, the pre-analytical rejection rates determined in these sample types were compared.

Results: Complete blood count $(0.40 \%)$ in emergency patients, $\mathrm{HbA}_{1} c(0.78 \%)$ in outpatients, biochemistry $(0.62 \%)$ in inpatients, hormones $(0.29 \%)$, urine $(6.19 \%)$ blood gases (1.03\%), coagulation (1.26\%), ESR (3.23\%) in critical patients, sample types had the highest pre-analytical rejection rate.

Conclusions: The source of causes that affect pre-analytical rejection rates, such as hemolyzed sample, clotted sample, or insufficient sample, may be due to the patient's bed rest, critical or emergency. An underlying disease, treatment, or frequent phlebotomy may also be a factor. The source of the causes that affect the pre-analytical rejection rates, such as incorrect request, incorrect registration, and incorrect tube, can usually be attributed to non-laboratory healthcare personnel.

Keywords emergency, inpatient, intensive care, outpatient, pre-analytical rejection rates

\section{INTRODUCTION}

The report, prepared by a group to be submitted to the Centers for Disease Control and Prevention in the USA, emphasized the conclusion that the laboratory was a fundamental 
element of the medical health system and an integral part of clinical decisions. ${ }^{1}$ Laboratory findings are effective in approximately $70 \%$ of these clinical decisions. ${ }^{2}$ Additionally, laboratory findings constitute $40-94 \%$ of all objective health record data. Therefore, the accuracy of laboratory results is critical for the diagnosis, treatment, monitoring, and follow-up of diseases. $^{3}$

The total testing process is divided into three stages: pre-analytical, analytical, and postanalytical. ${ }^{4}$ The pre-analytical phase includes the initial procedures of the testing process performed by healthcare professionals outside the laboratory walls and outside the direct control of the clinical laboratory. ${ }^{5}$ The analytical stage is the process that includes pretreatment and analysis of samples, and validation of results for samples occurring within the laboratory walls. The post-analytical stage refers to the process from the confirmation of laboratory test results to the use of the result for the benefit of the patient. ${ }^{6}$

Reasons for sample rejection in the laboratory usually occur at the pre-analytical stage. ${ }^{7}$ At this stage, which lasts until the sample acceptance in the laboratory, the travel process of the samples coming from the clinics is the most prone to sample rejection. ${ }^{8}$ Sample rejections at this stage occur during patient preparation, sample collection, sample handling, sample preparation, and sample storage. ${ }^{9}$ Sample rejections in this pre-analytical stage cover up to $70 \%$ of all sample rejections made in obtaining laboratory results. ${ }^{10}$ These sample rejections relate to procedures performed outside the laboratory walls. It is also caused by health personnel who are not under the direct control of the clinical laboratory. ${ }^{11}$

The samples sent to the laboratory for analysis come from patients whose health status may be very different. The health status of these patients may be urgent or may be nonurgent. Additionally, it may be a health status that needs to be hospitalized or it may be a health status that needs to be treated in the intensive care. This study aimed to evaluate the reasons for pre-analytical rejection rate detected in sample types taken from patients with different health status (emergency, outpatient, inpatient and critical). This evaluation process was done by comparing the pre-analytical rejection rates of sample types observed in these different health-status patient groups. In addition, analysis of sample type preanalytical rejection rates was performed in these patient groups. On the other hand, the reasons for pre-analytical rejection rates were examined in these sample types. With this evaluation, it was examined how the health status of the patient affected the formation of the pre-analytical rejection rates observed in different sample types.

\section{MATERIALS AND METHODS}

\section{Study design}

The type of study was a retrospective study. The study was conducted in Samsun, Turkey. The ethics committee decision required for the study was approved by the human research ethics committee of Samsun Training and Research Hospital on 26/02/2020. All study procedures were performed by human rights and complied with the principles of the Declaration of Helsinki. The study includes data from studied samples in the central laboratory 
(emergency, routine) of Health Science University, Samsun Training and Research Hospital in 2019. The total sample size included in this study was 1307013. Samples coming from the blood collection unit were evaluated in the sample acceptance unit, and the appropriate samples were accepted. On the other hand, unsuitable samples were rejected after being evaluated within the scope of pre-analytical reject reasons in the sample acceptance unit, and the rationale was entered into the laboratory information system. Samples with pre-analytical reject reasons (hemolysis, clotted, etc.) detected by the technicians during the analysis were rejected and new samples were requested, and if analytical reject reasons were found, the samples were reworked. Only samples rejected due to pre-analytical reject rates were included in the study. As sample type, eight types of samples were included in the study. These were complete blood count, biochemistry, hormones, urine, blood gases, coagulation, ESR, and $\mathrm{HbA}_{1} c$.

\section{Group criteria}

The samples were divided into four groups considering the health status of the patients. The groups were:

1. Emergency patients: It includes patients with an emergency condition who apply to the emergency department of the hospital.

2. Outpatients: It includes the patients who do not have any emergency, come to the outpatient clinics of the hospital for examination, and were not hospitalized for their treatment.

3. Inpatients: It includes patients who need to be hospitalized for various reasons such as diagnosis, treatment, and surgery. Patients hospitalized in the intensive care department were excluded from this group.

4. Critically ill patients: It includes patients hospitalized in intensive care units for various reasons such as respiratory failure, need for mechanical ventilation, shock, and other organ failures.

\section{Statistical analysis}

Rejected samples at the hospital were categorized according to sample types (complete blood count, biochemistry, hormones, urine, blood gases, coagulation, ESR, $\mathrm{HbA}_{1} c$ ). These rejected sample types were categorized into groups according to the health status of the patients. The categorized data of the groups were compared with the Chi-square test. In addition, the percentage frequency distribution of rejected sample types in each group was examined. On the other hand, the percentage frequency distribution of the causes was 
examined in each sample type. Data analysis was performed using Microsoft Excel version 2016 (Microsoft, Redmond, USA) and Statistical Package for the Social Sciences for Windows 22.0 (IBM, New York, USA).

\section{RESULTS}

\section{Rejection rates in sample types}

During the year in which this study was conducted, $719(0.20 \%)$ of 351796 complete blood count samples, $978(0.30 \%)$ of 326489 biochemistry samples, $117(0.05 \%)$ of 238531 hormones samples, $268(0.21 \%)$ of 127299 urine samples, $481(0.52 \%)$ of 93093 blood gases samples, $492(0.54 \%)$ of 90584 coagulation samples, 262 (0.45\%) of 57751 ESR samples, 166 $(0.77 \%)$ of $21470 \mathrm{HbA}_{1} c$ samples requested for analysis from the biochemistry laboratory were rejected for various reasons. Among these sample types, the highest rejection rate was observed in $0.77 \% \mathrm{HbA}_{1} c$ samples and the lowest rejection rate was observed in hormones samples with $0.05 \%$. The rejection rates of these sample types in the groups were given in Table 1.

\begin{tabular}{|c|c|c|c|c|c|}
\hline Sample type & & Emergency & Outpatient & Inpatient & Critically ill \\
\hline \multirow[t]{3}{*}{ Complete blood count } & Sample (n) & 38554 & 188070 & 92636 & 32536 \\
\hline & Reject (n) & 155 & 238 & 250 & 76 \\
\hline & Reject (\%) & 0.40 & 0.13 & 0.27 & 0.23 \\
\hline \multirow[t]{3}{*}{ Biochemistry } & Sample (n) & 96480 & 130897 & 66116 & 32996 \\
\hline & Reject (n) & 64 & 345 & 413 & 156 \\
\hline & Reject (\%) & 0.07 & 0.26 & 0.62 & 0.47 \\
\hline \multirow[t]{3}{*}{ Hormones } & Sample (n) & 28452 & 190471 & 14799 & 4809 \\
\hline & Reject (n) & 46 & 26 & 31 & 14 \\
\hline & Reject (\%) & 0.16 & 0.01 & 0.21 & 0.29 \\
\hline \multirow[t]{3}{*}{ Urine } & Sample (n) & 13670 & 97132 & 14882 & 1615 \\
\hline & Reject (n) & 24 & 60 & 84 & 100 \\
\hline & Reject (\%) & 0.18 & 0.06 & 0.56 & 6.19 \\
\hline \multirow[t]{3}{*}{ Blood gases } & Sample (n) & 27180 & 6387 & 38676 & 20850 \\
\hline & Reject (n) & 112 & 51 & 103 & 215 \\
\hline & Reject (\%) & 0.41 & 0.80 & 0.27 & 1.03 \\
\hline \multirow[t]{3}{*}{ Coagulation } & Sample (n) & 23410 & 30138 & 29048 & 7988 \\
\hline & Reject (n) & 96 & 169 & 126 & 101 \\
\hline & Reject (\%) & 0.41 & 0.56 & 0.43 & 1.26 \\
\hline \multirow[t]{3}{*}{ ESR } & Sample (n) & 0 & 44039 & 12193 & 1519 \\
\hline & Reject (n) & 0 & 100 & 113 & 49 \\
\hline & Reject (\%) & 0.00 & 0.23 & 0.93 & 3.23 \\
\hline \multirow[t]{3}{*}{$\mathrm{HbA}_{1} c$} & Sample (n) & 0 & 19788 & 1408 & 274 \\
\hline & Reject (n) & 0 & 155 & 10 & 1 \\
\hline & Reject (\%) & 0.00 & 0.78 & 0.71 & 0.36 \\
\hline
\end{tabular}




\section{Sample types rejection rates of groups}

ESR and $\mathrm{HbA}_{1} c$ analysis were not performed in the emergency laboratory of the hospital where the study was conducted. The highest rejected sample rate in the emergency patient group was found in blood gases and coagulation samples with $0.41 \%$. The highest rejected sample rate in the outpatient group was found in blood gases samples with $0.80 \%$. The highest rejected sample rate in the inpatient group was found in ESR samples with $0.93 \%$. The highest rejected sample rate in the critically ill patient group was found in urine samples with $6.19 \%$ (Table 1).

\section{Comparison of sample types rejection rates of groups}

The highest rejection rate in complete blood count samples was found in the emergency patient group. It was also higher than the rate found in the outpatient group $(p<0.001)$. The highest rejection rate in biochemistry samples was found in the inpatient group. It was also higher than the rates found in the emergency and outpatient groups $(p<0.001)$ (Table 1).

The highest rejection rate in hormones samples was found in the critically ill patient group. It was also higher than the rate found in the outpatient group. $(p<0.001)$. The highest rejection rate in urine samples was found in the critically ill patient group. It was also higher than the rates found in the other groups. $(p<0.001)$. In addition, the rate found in the inpatient group was higher than the rate found in the outpatient group ( $p=0.02)$ (Table 1).

The highest rejection rate in blood gases samples was found in the critically ill patient group. It was also higher than the rates found in the emergency and inpatient groups $(p<0.001)$. In addition, the rate found in the outpatient group was higher than the rates found in the emergency and inpatient groups $(p<0.001)$. The highest rejection rate in coagulation samples was found in the critically ill patient group. It was also higher than the rates found in the emergency and outpatient groups $(p<0.001)$ (Table 1$)$.

The highest rejection rate in ESR samples was found in the critically ill patient group. It was also higher than the rates found in the inpatient and outpatient groups $(p<0.001)$. In addition, the rate found in the inpatient group was higher than the rate found in the outpatient group $(p<0.001)$. The highest rejection rate in $\mathrm{HbA}_{1} c$ samples was found in the outpatient group. It was also higher than the rates found in the critically ill patient group $(p<0.001)$. In addition, the rate found in the inpatient group was higher than the rate found in the critically ill patient group $(p<0.001)$ (Table 1$)$.

\section{Proportions of rejected samples in groups}

The highest rate among rejected samples was complete blood count with $31.19 \%$ in the emergency patient group, biochemistry with $30.16 \%$ in the outpatient group, biochemistry with $36.55 \%$ in the inpatient group, and blood gases sample with $30.20 \%$ in the critically ill group (Table 2). 
Table 2 Distribution of rejected samples in groups according to sample types (\%).

\begin{tabular}{lllll} 
Sample type & Emergency & Outpatient & Inpatient & Critically ill \\
Complete blood count & 31.19 & 20.80 & 22.12 & 10.67 \\
Biochemistry & 12.88 & 30.16 & 36.55 & 21.91 \\
Hormones & 9.26 & 2.27 & 2.74 & 1.97 \\
Urine & 4.83 & 5.24 & 7.43 & 14.04 \\
Blood gases & 22.54 & 4.46 & 9.12 & 30.20 \\
Coagulation & 19.32 & 14.77 & 11.15 & 14.19 \\
ESR & 0 & 8.74 & 10.00 & 6.88 \\
HbA $_{1} \boldsymbol{c}$ & 0 & 13.55 & 0.88 & 0.14 \\
\hline
\end{tabular}

\section{Proportions of major causes of rejected sample types}

The major reasons of the rejected samples were given in Table 3.

\begin{tabular}{|c|c|}
\hline Sample type & major causes \\
\hline Complete blood count & $\begin{array}{l}\text { clotted blood (54.10\%), insufficient sample (38.53\%), incorrect tube } \\
(5.15 \%)\end{array}$ \\
\hline Biochemistry & $\begin{array}{l}\text { hemolyzed blood }(58.69 \%) \text {, incorrect order }(24.44 \%) \text {, incorrect reg- } \\
\text { istration }(5.83 \%) \text {, insufficient sample }(5.73 \%)\end{array}$ \\
\hline Hormones & $\begin{array}{l}\text { hemolyzed blood }(41.88 \%) \text {, incorrect tube }(24.79 \%) \text {, insufficient } \\
\text { sample }(17.95 \%) \text {, incorrect order }(11.11 \%)\end{array}$ \\
\hline Urine & $\begin{array}{l}\text { insufficient sample }(88.06 \%) \text {, incorrect tube }(5.22 \%) \text {, incorrect order } \\
(4.48 \%)\end{array}$ \\
\hline Blood gases & $\begin{array}{l}\text { clotted blood }(90.23 \%) \text {, insufficient sample }(7.28 \%) \text {, incorrect tube } \\
(1.87 \%)\end{array}$ \\
\hline Coagulation & $\begin{array}{l}\text { clotted blood (56.10\%), insufficient sample (33.33\%), incorrect tube } \\
(6.50 \%)\end{array}$ \\
\hline ESR & $\begin{array}{l}\text { clotted blood }(58.78 \%) \text {, insufficient sample }(26.72 \%) \text {, incorrect tube } \\
(8.02 \%)\end{array}$ \\
\hline $\mathbf{H b A}_{1} \boldsymbol{c}$ & $\begin{array}{l}\text { incorrect order }(86.75 \%) \text {, incorrect registration }(6.02 \%) \text {, incorrect } \\
\text { tube }(6.02 \%)\end{array}$ \\
\hline
\end{tabular}

\section{DISCUSSION}

In this study, pre-analytical rejection rates detected in eight different sample types requested from patients coming to Training and Research Hospital were evaluated. This evaluation process was carried out considering the effect of the health status of the patients (emergency, outpatient, inpatient, and critically ill).

Intensive care departments were developed as a result of the polio epidemic of the 1950s when widespread mechanical ventilation was required. Since then, the importance of inten- 
sive care departments in supporting critically ill patients has been indisputable. On the other hand, intensive care departments make up only $10 \%$ of inpatient beds. However, the acute care cost of intensive care patients constitutes approximately $30 \%$ of the acute care costs of inpatients. ${ }^{10}$ Similarly, laboratory test requests are more common in intensive care departments compared to other hospitalized patients. ${ }^{11}$ While $0.66 \%$ of the patients who applied to the hospital during the year of this study were treated in the intensive care department, $7.85 \%$ of the laboratory test requests were requested from the intensive care department. Among inpatients, these rates were $20.75 \%$ and $27.55 \%$, respectively.

Laboratory test requests, which are common in the intensive care department, can harm the patient in a number of ways. First, frequent phlebotomy may cause anemia and increase the need for blood transfusion. ${ }^{12}$ In a study investigating the cause of red blood cell transfusion in the intensive care department, it was found that patients underwent phlebotomy between 40 and $70 \mathrm{~mL}$ per day, with an average total volume of more than $1 \mathrm{~L}$ during their stay in the intensive care department. ${ }^{13}$ In a study with data from 7273 patients, the total volume was found to be $213 \mathrm{ml}$. In addition, it was concluded that the blood volume taken for laboratory tests affects blood loss, red blood cell transfusion and anemia in intensive care patients. ${ }^{14}$ In addition, red blood cell transfusion may be associated with hemolysis. ${ }^{15,16}$ In this study, the critically ill patient group consisted of patients hospitalized in the intensive care unit. This group had the highest rejection rate in the hormones, urine, blood gases, coagulation, and ESR sample types. It has the second-highest rejected sample rate of the biochemistry sample type. The most common reason for rejection, both in these sample types and in other sample types, was the insufficient sample. On the other hand, the highest cause of rejection in hormones and biochemistry sample types was hemolyzed blood. Reducing laboratory test requests in the intensive care department may reduce these rejected sample rates.

In addition to the fact that frequent phlebotomy causes anemia, drugs administered in the intensive care department may have side effects that may lead to hemolytic anemia. ${ }^{17}$ In addition, critically ill patients have coagulation abnormalities such as thrombocytopenia, consumption of clotting factors, and less commonly, disseminated intravascular coagulation. Thrombocytopenia can be induced by numerous clinical events such as hemodilution resulting from transfusion due to massive blood loss. ${ }^{18}$ Medications, including drugs commonly used in the intensive care department and mechanical cardiac assist devices, have also been associated with inducing thrombocytopenia. It is characterized by an abnormally low platelet count caused by increased consumption of platelets and other coagulation factors and prolonged coagulation times. ${ }^{17}$ In this study, the blood gases sample type had the highest rate among the rejected samples in the critically ill patient group. The highest reason for rejection in blood gases sample type was clotted blood with $90.23 \%$. In the intensive care department, the source of both clotted blood and hemolyzed blood and other causes of rejection was often related to the critical health status of the patient.

In a study on the management of hemolyzed samples in clinical laboratories, it has been reported that the most common and serious pre-analytical reject reason was the hemolyzed sample. In addition, it was stated that the detection and management of hemolyzed sam- 
ples were heterogeneous and should be standardized. Because hemolysis can occur both in vivo and in vitro. Intravascular hemolysis (in vivo) was always associated with underlying pathology. It was always difficult for the laboratory to differentiate between in vivo and in vitro hemolysis. ${ }^{19}$ In a study on the hemolysis index, it was stated that the hemolysis rate of inpatient samples (5.61\%) was significantly higher than that of outpatient samples $(2.21 \%) .{ }^{20}$ In this study, the inpatient group was the group with the second-highest rejected sample rate. In the rejected samples in the inpatient group, the highest rejected sample rate belongs to the biochemistry sample type. In addition, the highest rejection rate in biochemistry samples was observed in the inpatient group. In the biochemistry sample type, the highest rejection reason was the hemolyzed sample. This reason for rejection may be related to the treatment received by the patient or underlying pathology. In the biochemistry sample type, the second and third highest reasons for rejection were incorrect order and registration. These reasons for rejection were usually due to the registration of the sample in the hospital information system or the lack of information.

In a study on thrombosis consultation in the hospital setting, it was stated that the consultation requests from inpatients were quite different from those requested in the outpatient clinic. While the outpatient clinic usually includes patients with suspected hypercoagulation, in inpatients, the cases are more acute and require rapid evaluation. ${ }^{21}$ In Virchow's triad, which was shown as the main cause of thrombosis, the first cause was stagnation, bed rest and inactivity. ${ }^{22}$ In this study, among the samples rejected in the inpatient group, the sample types with the second and third highest rates were complete blood count and coagulation sample types, respectively. In these sample types, the highest rejection reason was the clotted sample. The stagnation, bed rest and immobility observed in hospitalized patients may be the source of this rejected sample.

In a study on thrombosis consultation in the hospital setting, it was stated that it was generally requested in the outpatient clinic for patients with suspected hypercoagulation. ${ }^{21}$ In a study in which severely injured trauma patients were evaluated for hypercoagulation, it was stated that severely injured patients were more prone to hypercoagulation. ${ }^{23}$ In this study, the highest rejected sample rate in the emergency patient group was observed in blood gases and coagulation sample types. Among the rejected samples in the emergency patient group, the sample types with the highest rates were complete blood count, blood gases and coagulation sample types, respectively. In these three sample types, the highest rejection reason was the clotted sample. Hypercoagulability observed in emergency cases may be the most important source of this rejection.

In this study, the outpatient group was the group with the highest number of patients. On the other hand, the group with the lowest rejection rate was the outpatient group. In addition, the highest rejection rate in the glycosylated hemoglobin sample type was found in the outpatient group. The glycosylated hemoglobin sample type was often rejected for reasons such as incorrect request, incorrect registration, and incorrect tube. These reasons for rejection, which were caused by hospital staff, may be related to the high number of patients. 


\section{CONCLUSIONS}

The hemolyzed sample, clotted sample and insufficient sample, rejection reasons may be patient's health status. The patient's health status can sometimes be related to the underlying disease and sometimes to the patient's current situation. Sometimes, it may also be related to the treatment applied to the patient or frequent phlebotomy. Less frequent phlebotomy can be done to prevent this. In addition, according to the treatment applied to the patient, the stages of patient preparation, sample collection, sample transport, sample preparation and sample storage can be performed. The reasons for incorrect request, incorrect registration and incorrect tube rejection can generally be caused by the registration of the sample in the hospital information system or the lack of information. Training of phlebotomists and other hospital staff will help reduce pre-analytical rejection rates. It can also be caused by patient density. Better organization of patient preparation, sample collection, sample transport, sample preparation and sample storage phases, the work of experienced personnel, increasing the number of personnel working in parallel with the increasing workload, and up-to-date follow-up of these steps will also help reduce pre-analytical rejection rates.

\section{DECLARATIONS}

\section{Authors' contributions}

Conceptualization and Formal analysis: AK. Data curation: RA. Funding acquisition: N/A. Investigation, Methodology, Project administration, Resources, Software, Supervision, Validation, Visualization, Writing-original draft, review \& editing: AK and RA. The authors have reviewed and approved the final proof before publication.

\section{Conflict of interest}

The authors declared that there is no conflict of interest.

\section{Ethical approvals}

This study was approved by the human research ethics committee of Samsun Training and Research Hospital (Application No: KAEK 2020/5/8).

\section{Data availability}

The data that support the findings of this study are available from the corresponding author, upon reasonable request.

\section{Funding resources}

This study did not receive any fund. 


\section{REFERENCES}

1. Wolcott J, Schwartz A, Goodman C. The Value of Laboratory Medicine to Health Care. In: Laboratory Medicine: A National Status Report. The Lewin Group; 2008. p. 19-65. Available from: https://stacks.cdc.gov/view/cdc/30726.

2. Hallworth MJ. The ' $70 \%$ claim': what is the evidence base. Ann Clin Biochem. 2011;48:487-488. Available from: 10.1258/acb.2011.011177.

3. Sepulveda JL. Variation, Errors, and Quality in the Clinical Laboratory. In: Dasgupta A, Sepulveda JL, editors. Accurate Results in the Clinical Laboratory A Guide to Error Detection and Correction. Elsevier; 2013. p. 1-8. Available from: 10.1016/B978-012-415783-5.00001-3.

4. Hammerling JA. A review of medical errors in laboratory diagnostics and where we are today. Lab Med. 2012;43(2):41-44. Available from: 10.1309/ LM6ER9WJR1IHQAUY.

5. Plebani M. Quality indicators to detect pre-analytical errors in laboratory testing. Clin Biochem Rev. 2012;33(3):85-88.

6. Lundberg GD. Acting on significant laboratory results. JAMA. 1981;245(17):17621763. Available from: 10.1001/jama.1981.03310420052033.

7. Ozcan O, Gureser S. Sources of preanalytical errors and the role of training in error prevention. Dicle Med J. 2012;39(4):524-530. Available from: 10.5798/diclemedj. 0921.2012.04.0194.

8. Lippi G, Chance JJ, Church S. Preanalytical quality improvement: from dream to reality. Clin Chem Lab Med. 2011;49(7):1113-1126. Available from: 10.1515/CCLM. 2011.600.

9. Carraro P, Zago T, Plebani M. Exploring the Initial Steps of the Testing Process: Frequency and Nature of Pre-Preanalytic Errors. Clin Chem. 2012;58(3):638-642. Available from: 10.1373/clinchem.2011.175711.

10. Wenham T, Pittard A. Intensive care unit environment. Anaesth Crit Care Pain Med. 2009;9(6):178-183. Available from: 10.1093/bjaceaccp/mkp036.

11. Ezzie ME, Aberegg SK, Jr JMO. Laboratory testing in the intensive care unit. Crit Care Clin. 2007;23(3):435-465. Available from: 10.1016/j.ccc.2007.07.005.

12. Lee J, Maslove DM. Using information theory to identify redundancy in common laboratory tests in the intensive care unit. BMC Med Inform Decis Mak. 2015;15:5959. Available from: 10.1186/s12911-015-0187-x.

13. Corwin HL, Parsonnet KC, Gettinger A. RBC transfusion in the ICU. Is there a reason? Chest. 1995;108(3):767-771. Available from: 10.1378/chest.108.3.767.

14. Chornenki J, James NL, Barty TE, R. Blood loss from laboratory testing, anemia, and red blood cell transfusion in the intensive care unit: a retrospective study. Transfusion. 2020;60(2):256-261. Available from: 10.1111/trf.15649.

15. L'Acqua C, Bandyopadhyay S, Francis RO. Red blood cell transfusion is associated with increased hemolysis and an acute phase response in a subset of critically ill children. Am J Hematol. 2015;90(10):915-920. Available from: 10.1002/ajh.24119. 
16. Hod EA, Brittenham GM, Billote GB. Transfusion of human volunteers with older, stored red blood cells produces extravascular hemolysis and circulating nontransferrin-bound iron. Blood. 2011;118(25):6675-6682. Available from: 10.1182/ blood-2011-08-371849.

17. Mcevoy MT, Shander A. Anemia, bleeding, and blood transfusion in the intensive care unit: causes, risks, costs, and new strategies. Am J Crit Care. 2013;22(6):1-14. Available from: 10.4037/ajcc2013729.

18. Greinacher A, Selleng K. Thrombocytopenia in the intensive care unit patient. Hematology Am Soc Hematol Educ Program. 2010;2010:135-143. Available from: 10.1182/asheducation-2010.1.135.

19. Simundic AM, Baird G, Cadamuro J, Costelloe SJ, Lippi G. Managing hemolyzed samples in clinical laboratories. Crit Rev Clin Lab Sci. 2020;57(1):1-21. Available from: 10.1080/10408363.2019.1664391.

20. Liu D, Li Y, Huang Y. The prevalence of hemolysis - a survey using hemolysis index. Clinical Chemistry and Laboratory Medicine (CCLM). 2017;55(4):90-91.

21. Hambleton J, Leung LL, Levi M. Coagulation: consultative hemostasis. Hematology Am Soc Hematol Educ Program. 2002;Available from: 10.1182/asheducation-2002. 1.335 .

22. Lyman GH, Khorana AA. Cancer, clots and consensus: new understanding of an old problem. J Clin Oncol. 2009;27(29):4821-4826. Available from: 10.1200/JCO.2009. 22.3032 .

23. Differding JA, Underwood SJ, Van PY, Khaki RA, Spoerke NJ, Schreiber MA. Trauma induces a hypercoagulable state that is resistant to hypothermia as measured by thrombelastogram. Am J Surg. 2011;201(5):587-591. Available from: 10.1016/j. amjsurg.2011.01.012.

\section{AUTHOR BIOGRAPHY}

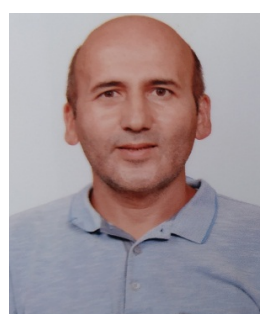

Adem Keskin got his Ph.D. in Biochemistry (Medicine) from Aydın Adnan Menderes University, Institute of Health Sciences, Department of Biochemistry (Aydın, Turkey). He currently works as a biologist at Aydın Gynecology and Pediatrics Hospital (Aydın, Turkey).

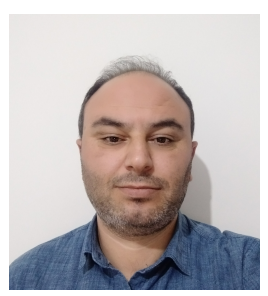

Recai Aci got his Ph.D. degree from 19 Mayis University, Institute of Health Sciences, Department of Biochemistry (Samsun, Turkey). He currently works as a biologist at Health Sciences University, Samsun Training and Research Hospital, Biochemistry Department (Samsun, Turkey). 